\title{
Effect of Oxcarbazepine on Serum Brain Derived Neurotrophic Factor in Bipolar Mania: An Exploratory Study
}

\author{
Rituparna Maiti ${ }^{1}$, Biswa Ranjan Mishra ${ }^{2}$, Jaseem Jowhar ${ }^{3}$, Debadatta Mohapatra ${ }^{2}$, Sansita Parida ${ }^{1}$, Debasis Bisoi ${ }^{1}$ \\ Departments of ${ }^{1}$ Pharmacology and ${ }^{2}$ Psychiatry, ${ }^{3}$ Medical Student, All India Institute of Medical Sciences (AllMS), Bhubaneswar, India
}

\begin{abstract}
Objective: In bipolar disorder, serum brain-derived neurotrophic factor (BDNF) level decreases leading to dysfunctions of critical neurotrophic, cellular plasticity and neuroprotective processes. The present study was conducted to evaluate the change in serum BDNF level with oxcarbazepine monotherapy in bipolar mania.

Methods: The present study is a prospective, interventional, open label clinical study conducted on 25 patients of bipolar mania and 25 healthy controls. Detailed history, clinical evaluation including Young Mania Rating Scale (YMRS) scoring and serum BDNF were assessed at baseline for all 50 subjects. The bipolar patients were prescribed tablet oxcarbazepine and followed up after 4 weeks for clinical evaluation and re-estimation of serum BDNF and YMRS scoring.

Results: The serum BDNF level in bipolar manic patients were compared with healthy controls at baseline and results revealed that there is a significant reduction $(p=0.002)$ in serum BDNF level in bipolar patients. At follow-up after 4 weeks, the mean change in serum BDNF in bipolar group who were on oxcarbazepine monotherapy was found statistically significant $(p=0.02)$ in comparison to healthy controls. In bipolar group, the YMRS score and serum BDNF at baseline have an inverse relation( $r=-0.59)$ whereas change of the YMRS score had a positive correlation ( $r=0.67)$ with the change of serum BDNF over 4 weeks. Conclusion: In bipolar mania serum BDNF level is low and it is found to be increased with short term monotherapy with oxcarbazepine.
\end{abstract}

KEY WORDS: Bipolar disorder; Brain-derived neurotrophic factor; Oxcarbazepine; Young Mania Rating Scale.

\section{INTRODUCTION}

Bipolar disorder (BD) is a chronic psychiatric illness of partially known pathophysiology which comprises, at a molecular and cellular level, imbalance of critical neurotrophic, neuroplasticity and resilience pathways and neuroprotective mechanisms. ${ }^{1)}$ The neurotrophic hypothesis postulates that mood disorders such as BD are associated with a lower expression of brain-derived neurotrophic factor (BDNF). Abnormalities of neurotrophins (NTs) and other trophic factors harmonize important variations which could be involved in the etiology of BD. As consistently reported in post-mortem studies, these modifications are generally associated with the disruption of distinct sub-regions and functions of the brain, one of which is the deregulation of NTs. ${ }^{2)}$

\footnotetext{
Received: August 9, 2016/ Revised: September 14, 2016

Accepted: October 6, 2016

Address for correspondence: Biswa Ranjan Mishra, MD Department of Psychiatry, All India Institute of Medical Sciences (AllMS), Bhubaneswar, Odisha 751019, India

Tel: +09776243691, Fax: +090674-2476002

E-mail: brm1678@gmail.com
}

NTs are capable of signaling neurons, glial cells and other cellular systems to enable survival, differentiation and growth. ${ }^{2-5)} \mathrm{BDNF}$ is one of the most studied and abundant NTs in the brain, which plays an important role in a variety of neural processes during the development of both animals and humans. Initially, BDNF is essential for neurogenesis, neuronal survival, and neural development pathways. In the adult, BDNF is not only crucial for synaptic plasticity and dendritic growth, but also for long-term memory consolidation. ${ }^{6}$ Previous studies have found serum BDNF to be significantly reduced in manic, hypomanic or depressive stages of $\mathrm{BD}$ in comparison to healthy controls. ${ }^{1,7-12)}$ Fernandes et al. ${ }^{13)}$ in their metaanalysis have concluded that BDNF is a potential biomarker of disease activity in $\mathrm{BD}$ whereas a recent meta-analysis by Munkholm et al. ${ }^{14)}$ could not conclude regarding the role of peripheral BDNF as a biomarker in $\mathrm{BD}$ and suggested further studies. de Sousa et al. ${ }^{15)}$ have shown a significant increase in serum BDNF levels after 4 weeks of lithium monotherapy in patients with acute bipolar mania and suggested its neuroprotective role which is attributed to its direct regulatory effect on BDNF. Oxcar-

(a) This is an Open-Access article distributed under the terms of the Creative Commons Attribution Non-Commercial License (http://creativecommons.org/licenses/by-nc/4.0) which permits unrestricted non-commercial use, distribution, and reproduction in any medium, provided the original work is properly cited. 
bazepine has demonstrated comparable efficacy to divalproate sodium and better tolerability profile in acute mania, ${ }^{16)}$ but Vasudev et al. ${ }^{17)}$ concluded in their meta-analysis that there is a need for adequately powered randomized controlled trials to assess therapeutic potential of oxcarbazepine across the spectrum of acute episodes in BD. Ghaemi et al. ${ }^{18)}$ found mild to moderate mood-stabilizing property of oxcarbazepine in refractory BD whereas Mosolov et al. ${ }^{19)}$ found that the efficacy of oxcarbazepine is comparable to carbamazepine in bipolar and schizoaffective disorder. Review of literature reveals that till date there is no study on the effect of oxcarbazepine on serum BDNF. The aim of the present study is to evaluate the change in serum BDNF level with oxcarbazepine monotherapy in bipolar mania and to compare the changes with healthy individuals.

\section{METHODS}

\section{Study Setting}

The study has been conducted on 25 patients of bipolar mania attending the outpatient department of psychiatry of All India Institute of Medical Sciences (AIIMS), Bhubaneswar, India, which is a tertiary care centre. The recruitment started from June 2015 and the study has been completed in December 2015.

\section{Study Population and Eligibility}

Forty patients aged 18 to 45 years, of either sex attending psychiatry outpatient department of AIIMS with bipolar mania (by International Classification of Diseases 10th revision, Diagnostic Criteria for Research [ICD-10 DCR]) were screened for the study. Schedules for Clinical Assessment in Neuropsychiatry (SCAN; ver. 2.1) was used for clinical assessment and diagnosis. ${ }^{20)}$ Initial diagnosis (by D.M.) was confirmed (by B.R.M.) and enrolment of 25 patients was done considering the inclusion and exclusion criteria. Another age and sex matched healthy subjects $(n=25)$ were recruited as control. Patients with the diagnosis of bipolar affective disorder (current episode mania) without psychotic symptoms with baseline Young Mania Rating Scale (YMRS) score $>20$ were included in the study. Recruited patients were either treatment naïve or treatment free for at least 4 weeks before inclusion. Patients with BD presenting during depressive/euthymic/mixed episode or patients with history of rapid cycling in the past 12 months were excluded. Patients with previous history of poor response to carbamazepine or oxcarbazepine; patients with comorbidities like psychotic disorder, organicity, substance abuse, personality disorder, intellectual disability and other neurotic disorders; pregnant and nursing women; patients with history of major medical or neurological illness were also excluded from the study. The subjects free from any medical and surgical illness, not taking any medication and YMRS score $<4$ were included under healthy control group.

\section{Study Design}

The proposed study is a prospective, interventional, parallel group clinical study and has been conducted in single center. Patients with BD were screened and 25 patients were enrolled following inclusion and exclusion criteria. After recruitment, detailed history was taken and mental status was evaluated. Severity of episode was assessed using YMRS and serum BDNF estimation was done at baseline. In the control arm, the age and sex matched healthy individuals $(n=25)$ were rated with YMRS and serum BDNF level was estimated at baseline. After baseline assessments, patients in test group were prescribed the tablet of oxcarbazepine $(10 \mathrm{mg} / \mathrm{kg}$ daily in two divided dose for one week followed by $15 \mathrm{mg} / \mathrm{kg}$ daily in two divided dose for next 3 weeks). Patients were given strict instruction not to take any other medication along with oxcarbazepine during the study period. All patients of test group were followed up after two weeks and four weeks. At two-week follow-up, drug compliance and clinical status including adverse drug reactions were checked. After four-weeks, all participants of both study groups were followed-up, YMRS was scored and serum BDNF level was estimated.

\section{Outcome Measures}

\section{Serum BDNF}

For estimation of serum BDNF, $3 \mathrm{ml}$ of venous blood was collected and allowed to clot by leaving it undisturbed at room temperature. The clotted blood was then centrifuged, serum was separated, collected in microcentrifuge tubes and stored in $-20^{\circ} \mathrm{C}$ refrigerator. Serum BDNF was estimated by enzyme-linked immunesorbent assay (ELISA) using human BDNF ELISA kit from Boster Biological Technology Co. Ltd. (Pleasanton, CA, USA). Boster's human BDNF ELISA Kit is based on standard sandwich ELISA technology.

\section{Young Mania Rating Scale (YMRS)}

YMRS, developed by R.C. Young in 1978, is one of the most frequently utilized 11-item clinician-rated rating 
scales to assess manic symptoms. The scale has 11 items and is based on the patient's subjective report of his or her clinical condition over the previous 48 hours. Additional information is based upon clinical observations made during the course of the clinical interview. There are four items that are graded on a 0 to 8 scale (irritability, speech, thought content, and disruptive/aggressive behavior), while the remaining seven items are graded on a 0 to 4 scale. These four items are given twice the weight of the others to compensate for poor cooperation from severely ill patients. ${ }^{21)}$

\section{Ethical Consideration}

A voluntary written informed consent was obtained from each subject after explaining the benefit and harm of joining the study and the freedom of withdrawing from the study at any moment they would like to. The study was conducted following Indian Council of Medical Research (ICMR)'s ethical guidelines for biomedical research on human subjects (2006) after getting approval of the institutional ethics committee (T/IM-NF/Pharm/14/19).

\section{Safety Evaluation}

The occurrence of adverse drug reactions was sought by nondirective questioning of the patient at follow-up visit. Patients had free access to the investigators for reporting any adverse effects experienced by them. There was provision for recording all adverse drug reactions, whether previously known or not, with their description, intensity, action taken, duration, outcome, and opinion about causal relationship to oxcarbazepine. At four weeks follow-up serum sodium level was estimated for all patients to look for hyponatremia (if any).

\section{Statistical Analysis}

Mann Whitney test and Wilcoxon signed rank test were used to compare the variables in study groups. Spearman's rank correlation coefficient (Spearman's $\rho$ ) was calculated for measuring correlation between YMRS score and serum BDNF. Statistical analyses was performed using statistical software Instat+ ver. 3.036 (Statistical Services Centre, University of Reading, Reading, England) considering a significance level of $p<0.05$.

\section{RESULTS}

Out of 40 bipolar mania patients screened, 12 patients were excluded as they did not meet the inclusion criteria and another three patients declined to participate. For recruitment of healthy controls, 35 subjects were screened and after exclusion of 10 subjects, 25 were included in the study. Total of 50 subjects were recruited in this study time and followed up after four weeks. In follow up, we have lost four bipolar patients and 21 patients were evaluated after drug therapy. In healthy control group, six subjects were lost and 19 subjects were evaluated at follow-up (Fig. 1).

The baseline demographic data and clinical characteristics of all 50 subjects participated in this study have been presented in the Table 1. This outpatient department based study has been conducted in our institute, which is a tertiary care center. All results/data produced by this study may not show similarity with other population-based studies because of the possibility of referral bias. Eighty percent of subjects were male, and mean age of the subjects was $34.3 \pm 9.6$ years. Mean age of onset and mean duration of suffering from bipolar mood disorder were $30.5 \pm 9.7$ years and $3.4 \pm 2.9$ years, respectively. Mean du-

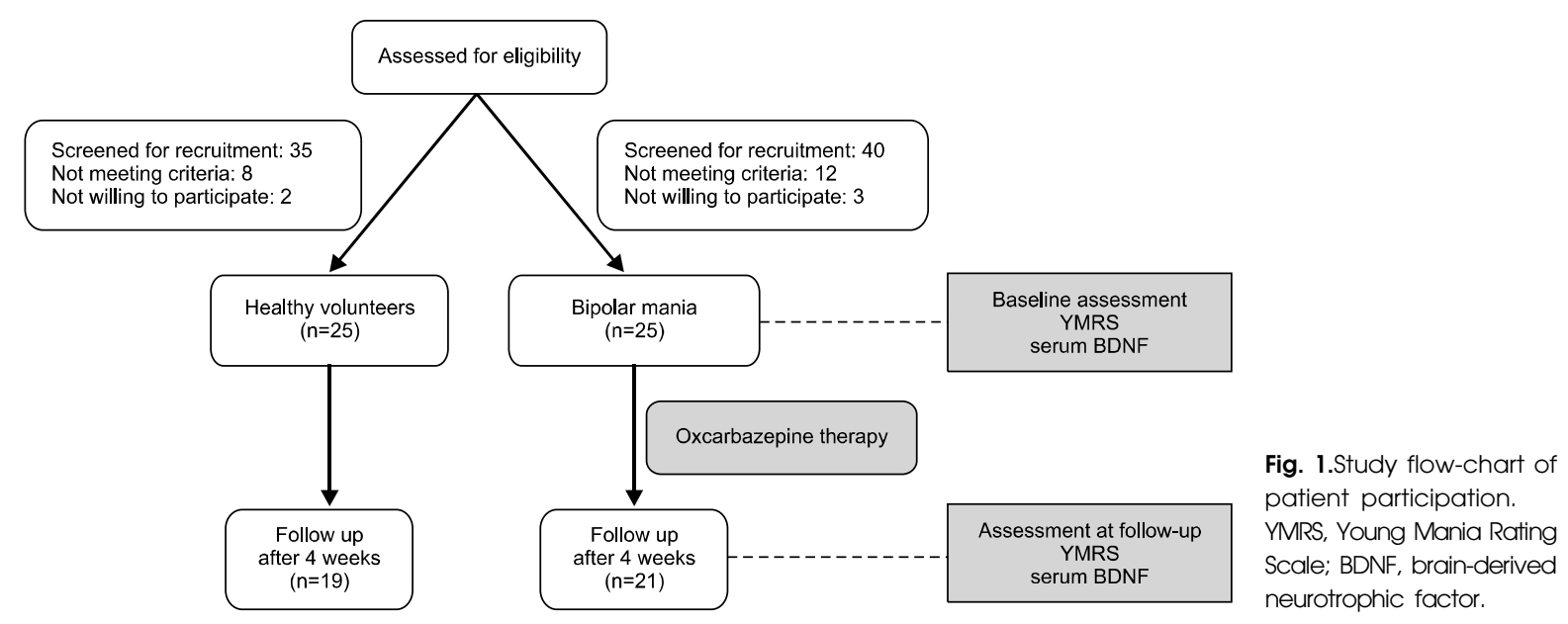


Table 1. Baseline demographic data and clinical characteristics

\begin{tabular}{|c|c|c|c|}
\hline Characteristic & Healthy control $(n=25)$ & Bipolar mania $(n=25)$ & $p$ value* \\
\hline Patients recruited $(n)$ & 25 & 25 & - \\
\hline Female sex (\%) & 20 & 20 & - \\
\hline Age $(y r)$ & $34.44 \pm 9.53$ & $34.16 \pm 9.89$ & 0.97 \\
\hline Duration of illness (yr) & - & $3.4 \pm 2.9$ & - \\
\hline Age of onset (yr) & - & $30.5 \pm 9.7$ & - \\
\hline Episodes $(n)$ & - & $3(1-4)$ & - \\
\hline Episode duration (mo) & - & $2.5 \pm 1.7$ & - \\
\hline Family history of psychiatric illness & - & $6 / 25(24)$ & - \\
\hline Young Mania Rating Scale score & $1.36 \pm 0.81$ & $31.1 \pm 7.2$ & $<0.0001^{\dagger}$ \\
\hline Serum BDNF (pg/ml) & $1,964.9 \pm 772.9$ & $1,365.6 \pm 335.3$ & $0.002^{\dagger}$ \\
\hline
\end{tabular}

Values are presented as number or percent only, mean \pm standard deviation, median (interquartile range), or number (\%).

*By Mann-Whitney test; ${ }^{\dagger}$ statistically significant.

Table 2. The change in serum BDNF over four weeks between patients with bipolar mania and healthy controls

\begin{tabular}{|c|c|c|c|c|c|}
\hline \multirow{2}{*}{ Serum BDNF (pg/ml) } & \multicolumn{4}{|c|}{ Serum BDNF (pg/ml) } & \multirow{2}{*}{$\begin{array}{l}\text { Difference between } \\
\text { the groups* }\end{array}$} \\
\hline & First visit & Second visit & Mean difference & $p$ value ${ }^{\S}$ & \\
\hline Healthy control group $(n=19)$ & $1,944 \pm 797.7$ & $1,920.9 \pm 783.2$ & $23.1^{\dagger}$ & 0.29 & $95 \% \mathrm{Cl},-124.45$ to -10.643 ; \\
\hline Bipolar mania group $(n=21)$ & $1,373.2 \pm 324.9$ & $1,463.9 \pm 256.5$ & $90.7^{\ddagger}$ & $<0.001^{* *}$ & $p^{\|}=0.02^{* *}$ \\
\hline
\end{tabular}

Values are presented as mean \pm standard deviation.

BDNF, brain-derived neurotrophic factor; $\mathrm{Cl}$, confidence interval.

*Healthy control vs. bipolar mania, ${ }^{\dagger}$ Healthy control, ${ }^{\ddagger}$ Bipolar mania.

§Wilcoxon Rank sum test, "Mann-Whitney test, ** statistically significant.

ration of manic episode was $2.5 \pm 2.7$ months. In our study, 15 patients $(60.0 \%)$ had onset of the disease before 35 years, out of which nine patients had onset before 25 years. In bipolar group, median number of manic episodes was found to be three. In $24 \%$ patients, there was positive family history of psychiatric disorders which reflects the strong genetic basis of the illness.

\section{Change in YMRS Score}

In both groups YMRS scoring was done at baseline and at four-weeks follow-up. The YMRS score among 21 follow-up bipolar manic patients was found to decrease from $30.1 \pm 7.3$ at baseline to $17.1 \pm 4.2$ after four weeks. The change in YMRS score among bipolar mania patients was found to be significant $(p<0.001)$ but there was no significant change $(1.42 \pm 0.84$ to $1.63 \pm 0.76, p=0.34)$ in YMRS score among healthy controls.

\section{Change in Serum BDNF}

At baseline: Mean Serum BDNF level among healthy controls was $1,964.9 \mathrm{pg} / \mathrm{ml}$ and in bipolar patients it was found to be $1,365.6 \mathrm{pg} / \mathrm{ml}$. The difference in serum BDNF level at baseline was found to be statistically significant $(p=0.002)$ (Table 1).
Change in serum BDNF in bipolar group

Among 21 follow-up patients, mean serum BDNF level was $1,373.2 \mathrm{pg} / \mathrm{ml}$ at baseline and the level was found to be increased to $1,463.9 \mathrm{pg} / \mathrm{ml}$ at follow-up after four weeks of oxcarbazepine therapy. The change in serum BDNF in bipolar patients was statistically significant ( $p$ $<0.001$ ) (Table 2).

\section{Change in serum BDNF in healthy controls}

Among 19 follow-up subjects, mean serum BDNF level was $1,944 \mathrm{pg} / \mathrm{ml}$ at baseline and the level was found to be $1,920.9 \mathrm{pg} / \mathrm{ml}$ at follow-up after four weeks. The change in serum BDNF in healthy controls was not statistically significant $(p=0.29)$ (Table 2$)$.

\section{Comparison of mean change in serum BDNF between study groups}

The mean changes of serum BDNF in both groups were compared by Mann-Whitney test and the change in bipolar mania group was found to be statistically significant $(p=0.02)$ (Table 2).

\section{Correlation between YMRS Score and Serum BDNF}

The correlation between two variables (YMRS score 


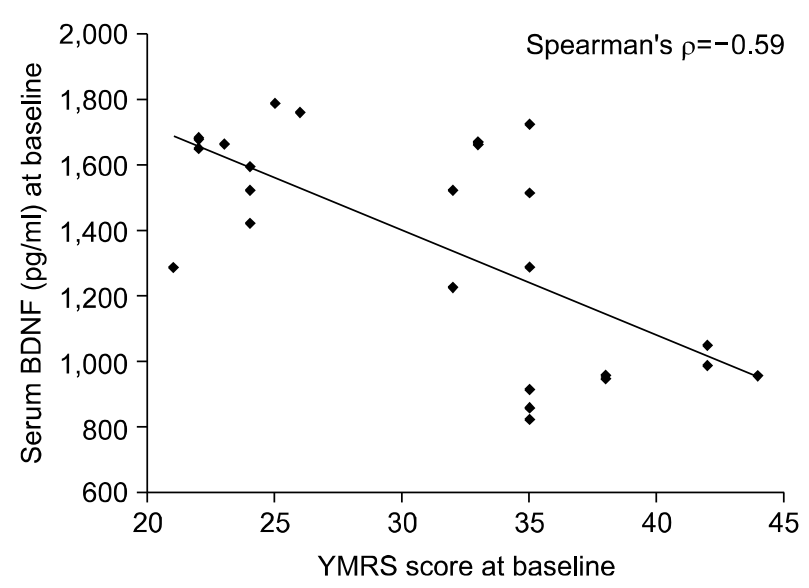

Fig. 2. The correlation between Young Mania Rating Scale (YMRS) score and serum brain-derived neurotrophic factor (BDNF) level in patients with bipolar mania.

and serum BDNF at baseline) was assessed using Spearman's rank correlation coefficient (Spearman's $\rho$ ). In bipolar group, the YMRS score and serum BDNF at baseline have an inverse relation (Spearman's $\rho=-0.59$ ) whereas change of the YMRS score had a positive correlation (Spearman's $\rho=0.67$ ) with the change of serum BDNF over four weeks. The correlation has been represented in Figures 2 and 3.

\section{Safety Evaluation}

Five patients reported mild headache, nausea and drowsiness during follow-up at second week. The drug was not discontinued because of mild degree of side effects. In the test group at follow-up, serum sodium levels were found to be within normal range without any report of hyponatremia.

\section{DISCUSSION}

The results of YMRS scoring showed a significant decrease suggesting improvement in symptoms in all bipolar patients. Oxcarbazepine has been already used in mania and in this study also significant improvement in YMRS score suggests the anti-manic effect of oxcarbazepine. The serum BDNF level in bipolar manic patients were compared with healthy controls at baseline and the results revealed that there is significant decrease in serum BDNF level in bipolar patients. In BD, lower BDNF levels have been described in both depressive and manic episodes and our present study supports the findings of the previous studies. ${ }^{9,22-24)}$ This may be attributed to the decreased hippocampal BDNF, proBDNF and $\mathrm{p} 75$ receptor protein expression in BD as revealed in the study by Dunham et al. ${ }^{25)}$ The severity of mania was found to have a negative corre-

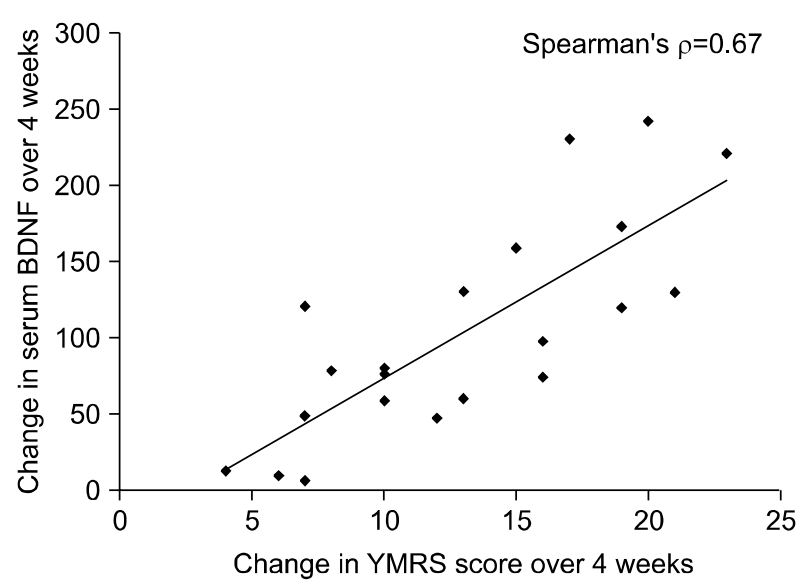

Fig. 3. The correlation between the change of Young Mania Rating Scale (YMRS) scores and the change in serum brainderived neurotrophic factor (BDNF) level in patients with bipolar disorder.

lation with serum BDNF levels which supports the neurotrophic hypothesis of BD. Machado-Vieira et al. ${ }^{22)}$ also showed an inverse association with severity of manic symptoms in their study.

In the bipolar group, the baseline serum BDNF level was decreased in comparison to healthy controls. But after four weeks treatment with oxcarbazepine, there was a significant increase in serum BDNF level. To best of our knowledge, this is the first study to evaluate the effects of oxcarbazepine monotherapy on serum BDNF levels in bipolar mania and our study reveals the fact that oxcarbazepine has a favorable effect on serum BDNF level suggesting its possible neurotrophic effect in BD. Another important finding of this study was the correlation between change in YMRS score and change in serum BDNF in bipolar patients. We found a significant positive correlation between the two variables suggesting a directly proportionate relationship between increase in serum BDNF level with symptomatic improvement in BD. Till date only lithium has been evaluated for its effect on serum BDNF and it was concluded that lithium monotherapy significantly increased plasma BDNF levels after four weeks but there was no significant correlation was found between changes in YMRS scores and BDNF levels. ${ }^{15)}$ From this aspect, oxcarbazepine monotherapy in bipolar mania shows difference from lithium monotherapy, therefore a head-to-head comparative study can answer the question of superiority. In the present study it has been observed that after four weeks oxcarbazepine therapy, serum BDNF has improved but still significantly lower $(p=0.016)$ than the healthy control. This finding suggests that a longer duration of treatment might be necessary for normalization 
of serum BDNF level.

Till date there is no animal study or in-vitro study related to the effect of oxcarbazepine on BDNF and its mechanistic details. Chang et $_{\text {al. }}{ }^{26)}$ found that chronic carbamazepine and lamotrigine significantly increased BDNF mRNA and protein levels in the frontal cortex. Mai et al $^{27)}$ has studied BDNF-mediated signal transduction and its modulation by glycogen synthase kinase 3 beta (GSK $3 \beta$ ) and mood stabilizing agents. They found that BDNF increased the phosphorylation of the fork head transcription factor FKHRL1 through activation of the phosphatidylinositol 3-kinase (PI3K)/Akt signaling pathway, and the phosphorylation of the cyclic AMP response element binding protein (CREB) through activation of extracellular signal-regulated kinase1/2 (ERK1/2). BDNF also increased serine (9) -phosphorylation of GSK3 $\beta$, which inhibits GSK3 $\beta$ activity. Over expression of GSK3 $\beta$ did not affect BDNF-induced phosphorylation of Akt, ERK1/2, or FKHRL1, but abolished CREB phosphorylation induced by BDNF. Carbamazepine was found to induce a rapid and prolonged phosphorylation of ERK $1 / 2$ and CREB in the absence or the presence of BDNF. Therefore, increased GSK3 $\beta$ selectively attenuates BDNF-induced CREB phosphorylation and carbamazepine can facilitate activation of CREB. ${ }^{27)}$ Oxcarbazepine being structurally analogous to carbamazepine, may modulate the signaling pathway in a similar fashion to increase BDNF level.

The main limitations of the study are small sample size, lack of active comparator group, a short follow up period of 4 weeks, estimation of only one NT and plasma level of oxcarbazepine has not been estimated. Longer follow-ups are required to comment on the sustained and adequate effect of oxcarbazepine therapy on serum BDNF level in BD.

In conclusion, analysis of results of all the parameters of the present study indicate that in bipolar mania serum BDNF is decreased which can be increased by oxcarbazepine monotherapy and symptomatic improvement has a positive correlation with the change of serum BDNF. Because small sample size, lack of active comparator group, and short follow-up period were main limitations, the findings of this study can be confirmed by a multicentric, randomized, double-blind, large population based study.

\section{Acknowledgments}

The authors would like to express heartfelt thanks to Dr. Debasish Hota, D.M., Dr. Biswa Mohan Padhy, D.M., and Dr. Zubair Ahmed Karim, PhD, for their valuable suggestions, active help, and encouragement.
This study was registered as follows: Registry name: ClinicalTrials.gov; URL: https:/clinicaltrials.gov/ct2/show/ record/NCT02456896; Registration number: NCT02456896; WHO ICTRP: http://apps.who.int/trialsearch/Trial2.aspx? TrialID $=$ NCT02456896.

\section{REFERENCES}

1. Frey BN, Andreazza AC, Houenou J, Jamain S, Goldstein BI, Frye MA, et al. Biomarkers in bipolar disorder: a positional paper from the International Society for Bipolar Disorders Biomarkers Task Force. Aust N Z J Psychiatry 2013;47:321-332.

2. Mufson EJ, Kroin JS, Sendera TJ, Sobreviela T. Distribution and retrograde transport of trophic factors in the central nervous system: functional implications for the treatment of neurodegenerative diseases. Prog Neurobiol 1999;57:451484.

3. Kaplan DR, Miller FD. Neurotrophin signal transduction in the nervous system. Curr Opin Neurobiol 2000;10:381-391.

4. Huang EJ, Reichardt LF. Neurotrophins: roles in neuronal development and function. Annu Rev Neurosci 2001;24:677736.

5. Poo MM. Neurotrophins as synaptic modulators. Nat Rev Neurosci 2001;2:24-32.

6. Post RM. Role of BDNF in bipolar and unipolar disorder: clinical and theoretical implications. J Psychiatr Res 2007; 41:979-990.

7. Hashimoto K, Shimizu E, Iyo M. Critical role of brainderived neurotrophic factor in mood disorders. Brain Res Brain Res Rev 2004;45:104-114.

8. Karege F, Bondolfi G, Gervasoni N, Schwald M, Aubry JM, Bertschy G. Low brain-derived neurotrophic factor (BDNF) levels in serum of depressed patients probably results from lowered platelet BDNF release unrelated to platelet reactivity. Biol Psychiatry 2005;57:1068-1072.

9. Cunha AB, Frey BN, Andreazza AC, Goi JD, Rosa AR, Gonçalves CA, et al. Serum brain-derived neurotrophic factor is decreased in bipolar disorder during depressive and manic episodes. Neurosci Lett 2006;398:215-219.

10. Sen S, Duman R, Sanacora G. Serum brain-derived neurotrophic factor, depression, and antidepressant medications: meta-analyses and implications. Biol Psychiatry 2008;64: 527-532.

11. Fernandes BS, Gama CS, Ceresér KM, Yatham LN, Fries $\mathrm{GR}$, Colpo G, et al. Brain-derived neurotrophic factor as a state-marker of mood episodes in bipolar disorders: a systematic review and meta-regression analysis. $J$ Psychiatr Res 2011;45:995-1004.

12. Ray MT, Shannon Weickert C, Webster MJ. Decreased $B D N F$ and TrkB mRNA expression in multiple cortical areas of patients with schizophrenia and mood disorders. Transl Psychiatry 2014;4:e389.

13. Fernandes BS, Molendijk ML, Köhler CA, Soares JC, Leite CM, Machado-Vieira R, et al. Peripheral brain-derived neurotrophic factor (BDNF) as a biomarker in bipolar disorder: a meta-analysis of 52 studies. BMC Med 2015; 13:289.

14. Munkholm K, Vinberg M, Kessing LV. Peripheral blood brain-derived neurotrophic factor in bipolar disorder: a comprehensive systematic review and meta-analysis. Mol Psychiatry 2016;21:216-228.

15. de Sousa RT, van de Bilt MT, Diniz BS, Ladeira RB, Portela 
LV, Souza DO, et al. Lithium increases plasma brainderived neurotrophic factor in acute bipolar mania: a preliminary 4-week study. Neurosci Lett 2011;494:54-56.

16. Kakkar AK, Rehan HS, Unni KE, Gupta NK, Chopra D, Kataria D. Comparative efficacy and safety of oxcarbazepine versus divalproex sodium in the treatment of acute mania: a pilot study. Eur Psychiatry 2009;24:178-182.

17. Vasudev A, Macritchie K, Vasudev K, Watson S, Geddes J, Young AH. Oxcarbazepine for acute affective episodes in bipolar disorder. Cochrane Database Syst Rev 2011;(12): CD004857.

18. Ghaemi SN, Ko JY, Katzow JJ. Oxcarbazepine treatment of refractory bipolar disorder: a retrospective chart review. Bipolar Disord 2002;4:70-74.

19. Mosolov SN, Kostiukova EG, Ladyzhenskiǐ MIa. [Comparative efficacy and tolerability of carbamazepine and oxcarbazepine during long therapy of patients with bipolar and schizoaffective disorders]. Zh Nevrol Psikhiatr Im S S Korsakova 2009;109:36-41. Russian.

20. Wing JK, Babor T, Brugha T, Burke J, Cooper JE, Giel R, et al. SCAN. Schedules for clinical assessment in neuropsychiatry. Arch Gen Psychiatry 1990;47:589-593.

21. Young RC, Biggs JT, Ziegler VE, Meyer DA. A rating scale for mania: reliability, validity and sensitivity. $\mathrm{Br} J$ Psy- chiatry 1978;133:429-435.

22. Machado-Vieira R, Dietrich MO, Leke R, Cereser VH, Zanatto V, Kapczinski F, et al. Decreased plasma brain derived neurotrophic factor levels in unmedicated bipolar patients during manic episode. Biol Psychiatry 2007;61: 142-144.

23. Lin PY. State-dependent decrease in levels of brain-derived neurotrophic factor in bipolar disorder: a meta-analytic study. Neurosci Lett 2009;466:139-143.

24. Rabie MA, Mohsen M, Ibrahim M, El-Sawy Mahmoud R. Serum level of brain derived neurotrophic factor (BDNF) among patients with bipolar disorder. J Affect Disord 2014;162:67-72.

25. Dunham JS, Deakin JF, Miyajima F, Payton A, Toro CT Expression of hippocampal brain-derived neurotrophic factor and its receptors in Stanley consortium brains. $J$ Psychiatr Res 2009;43:1175-1184.

26. Chang YC, Rapoport SI, Rao JS. Chronic administration of mood stabilizers upregulates BDNF and bcl-2 expression levels in rat frontal cortex. Neurochem Res 2009;34: 536-541.

27. Mai L, Jope RS, Li X. BDNF-mediated signal transduction is modulated by GSK3beta and mood stabilizing agents. $J$ Neurochem 2002;82:75-83. 